\title{
IDENTIFYING DIFFICULTIES IN THE KNOWLEDGE TRANSFER PROCESS IN GLOBAL SOFTWARE: A QUALITATIVE ANALYSIS
}

\author{
Franciney O. Lima, Victoria Carolina S. Mattos, Raquel F. V. Cunha, Alexandre S. Teixeira, \\ Marcelo S. Ayres, Luis H. Pascareli, Fernando B. Pires and Bruno A. Bonifácio \\ Sidia Institute of Science and Technology \\ Manaus - Amazonas, Brazil
}

\begin{abstract}
Due to increasing demand for new technologies, it is common in software projects to be necessary to incorporate new team members. However, when new hires join a company, they should learn about working process, organization structure, and company corporate culture. Therefore, companies should be adopting strategies to manage and transfer knowledge to better support newcomers. Sidia R\&D Institute had to hire new members to increase our Project Leader team, consequence of a massive project demand. As we work in a distributed environment, we use Wiki as an alternative tool for sharing knowledge and for helping newcomers learn about our software development process. However, newer members reported great difficulty during Wiki usage. This paper reports results of a qualitative study aimed to identify the difficulties faced by new team members during wiki usage at Sidia R\&D Institute, as well as identify actions that are often adopted to mitigate these difficulties. The study reveals that despite of wiki being important to improve knowledge transfer in Sidia team, newly PLs had difficulties to access wiki information caused by usability issues and lack of adequate knowledge management.
\end{abstract}

\section{KEYWORDS}

Qualitative Analysis, Grounded Theory, Knowledge Management, Knowledge Sharing

\section{INTRODUCTION}

As software development is increasingly globalized, it is common from massive, in software projects, incorporate new team members. However, newcomers, especially new hires, usually need more time to become acquainted with projects (Bao et al., 2019). During onboarding period, they face several difficulties and challenges such as misunderstandings process, learning curve, and other issues that cause expectation breakdowns (Avram, 2007). For this reason, knowledge management process must be organized and implemented in order to reduce technical and sociological difficulties.

In this context, companies have been adopting varying strategies to manage and transfer knowledge since, success can be difficult (Heredia et al., 2017). To better support newcomers in onboarding process, difficulties must be identified and understood.

Sidia is a R\&D Institute located in Brazil that works collaborating with Samsung Mobile division, located in South Korea, and external stakeholders. Resulting from massive project demand, Sidia had to hire new members to increase team responsible to manage software development process, the Project Leader (PL) team. Despite initial training, new members still faced difficulties, even with the transfer of knowledge from more experienced colleagues to new members, while they are preparing for a project. This scenario generated great difficulty for knowledge management. To reduce these difficulties, we used Wiki as an alternative for sharing knowledge and for helping newcomers learn about our software development process. However, newer PLs reported great difficulty during Wiki usage, due to a large amount of information and difficult to use and explore wiki content.

This paper presents a qualitative analysis aimed to understand which knowledge transfer difficulties are faced by newer PLs. In previous work, we identified alternatives to improve knowledge management process and we identified usability issues of wiki (Cunha et al., 2020). In this paper, we aimed to identify newcomers' 
learning process and show suggestions applied to improve documentation on Wiki. To accomplish it, we use Grounded Theory (GT) procedures to better understand data results. Thus, we hope to assist companies to improve knowledge transfer and access information in GSD environment.

This paper is structured as follows: Section 2 provides some related work and description of our Company Sidia as well as the GSD setting where we are in. Section 3 describes study planning and design, which we describe how we conducted the survey, and briefly the previous study. In Section 4, we present results achieved. Section 5 concludes and shows some future directions we are planning to follow to continue improving onboarding process and knowledge transfer in the company.

\section{BACKGROUND}

Due to distributed settings, raises the question of how to speed up the learning of project's newcomers (Kroll et al. 2016). To some extent, newcomers usually need to learn social and technical aspects alone, exploiting existing information in mailing lists, source code repositories, and issue manager (Mahmood, 2015). Furthermore, newcomers may not receive enough training or may not have intimate knowledge of the practices they are normally trained to follow (Heredia et al.,2017).

In this context, tools are essential for collaboration among team members, enabling communication, and knowledge management with more effectiveness (Kanakis, 2019). For this reason, some tools can support as much communication, coordination, documentation as knowledge management, especially in GDS environment.

In this sense, several investigations have focused on understanding how tools can improve collaboration, communication and knowledge management. Due to cost reduction, companies have been adopting Wiki as alternative for knowledge management (Smite, 2017; Bao et al. 2019; Portillo-Rodriguez, 2012). According to related works, Wiki is important resource to knowledge management. However, when Wiki has several information, users can face difficulty to use and this scenario can be a problem during onboarding process.

Sidia is a R\&D Institute, responsible for improvements on the Android Platform of Samsung products in all Latin America. Institute works collaborating with Samsung Mobile division, located in Korea, and external stakeholders provided by Mobile Network Operators (MNO) from other Latin countries (e.g., Brazil, Mexico, Chile, Peru). Each software project is managed by one PL, responsible to lead developers' team, managing requirements of each software version, negotiate deadlines, and update schedules for each project.

As we work in a distributed environment, we use Wiki as an alternative for sharing knowledge. In Wiki, we put information about working process, tools used, focal points of each MNO, stages of software development process etc. However, we observed that novice PLs faced great difficulty in using the Wiki due to a large amount of information, and difficulty to use and explore information access.

For this reason, we performed a survey with newcomers from our team. Our previous work relied on difficulties faced by newcomers to search for work process information in Wiki and show quantitative results considering perceived usefulness and perceived ease of use indicators within the Technology Acceptance Model - TAM (Cunha et al., 2020). Next Section presents the research conducted, findings, and how we use results to improve our Wiki pages.

\section{IMPROVING WIKI THROUGH QUALITATIVE ANALYSIS}

Due to massive projects demand, Sidia had to hire new members to increase headcount of PL team. As we work in GSD environment, initial trainings were not enough during onboarding period, hence newly PLs did not have knowledge of project management practices in company.

For this reason, we have been adopting Wiki as alternative tool to manage knowledge. In Wiki tool we have information since overview process until tutorials about tools usage and other working best practices. However, our team had strong difficult to use Wiki content information. In this case, we performed a survey using online questionnaire to understand difficulties faced by our newcomers. Thus, we design a study to analyze data collected. The previous study was detailed reported at Cunha et al. (2020). In this paper we will present qualitative results and improvements applied as contribution of previous work. 


\subsection{Quantitative Results}

The goal of this study was to evaluate Wiki content to knowledge transfer from the perspective of new project leaders by Sidia. In this scenario we designed the study, to collect data using an online questionnaire.

Table 1. Previous study results (Cunha et al., 2020)

\begin{tabular}{ll}
\hline Experimental Design & \multicolumn{1}{c}{ Summary Results } \\
\hline Participants & $\begin{array}{l}\text { We choose 24 volunteer participants of PL team. This participants } \\
\text { were newly and have initial experience on Sidia project process. }\end{array}$ \\
& $\begin{array}{l}\text { To evaluate quality of use and acceptance when participants interacted } \\
\text { with wiki, we have analyzed perceived usefulness and perceived ease } \\
\text { of use indicators within Technology Acceptance Model - TAM. This } \\
\text { model is focused on aspects that are strongly correlated to user }\end{array}$ \\
& acceptance of a given technology. \\
Results & $\begin{array}{l}\text { During study execution, we have applied a questionnaire asking for } \\
\text { subjects' opinions towards their improvement. We available } \\
\text { questionnaire during a week. We decide to collect information about } \\
\text { frequency of usage Wiki due to understanding if information enabled } \\
\text { in Wiki is adequate and useful to assist newcomers. Considering ease } \\
\text { of use, 42\% reported that they have difficulty with Wiki content } \\
\text { presentation, and another 32\% consider Wiki ease to find information. } \\
\text { Considering useful perception, } 76 \% \text { of users reported as positive } \\
\text { results. }\end{array}$ \\
\hline
\end{tabular}

Quantitative results were useful to identify difficulties, failures on training faced by newly PLs. In addition, we also decided to collect qualitative data regarding the participants' interaction with the Wiki. We made such decision aiming to obtain a more accurate result. Thus, we would be able to combine quantitative and qualitative data to better understand the identified problems. Next section we present qualitative analysis.

\subsection{Qualitative Results}

We gathered data from the follow-up questionnaire from each subject. We intended that questionnaires' data could assist us identifying and understanding which features could be improved to facilitate knowledge transfer, as well as which problems could be compromising the learnability. To analyze data collected we use procedures of Grounded Theory - GT (Mills et al. 2006). We use this method to build knowledge about improvements in Wiki and to identify relationship between subjects reported and some items.

We extracted qualitative data and coding using data reported by PLs. Then, codes found in questionnaires were grouped according to their properties, thus forming concepts that represent categories. For the analysis of inspector's interaction with technique, following categories were defined: Learning Strategies, Wiki Issues, Improvements and Ideas. Considering Learning Strategies category, we found important results to understand how newly PLs learning working process, see Figure 1.

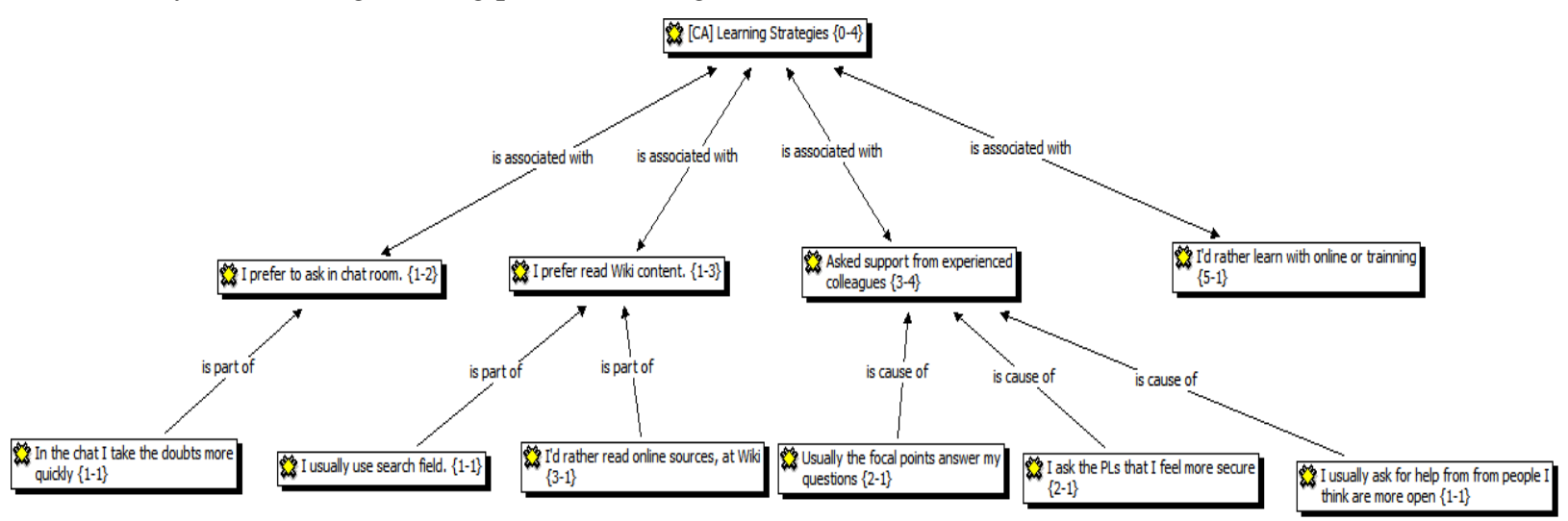

Figure 1. Points related learning strategies follow by newly PLs 
About learning strategies, we identified four interactions category used by PLs. Some PLs related that prefer to learn with online or training. Some PLs prefer to ask for experienced PLs on chatroom. One participant related that "In the chat I take the doubts more quickly". Another PLs prefer to read Wiki content using resources available in the tool. One participant said that "I usually use search field", an others participants related that "I'd rather read online sources, at Wiki". In addition, most participants prefer to ask support for experienced colleagues. Some participants related that focal points of project have important role to support newly PL, as "Usually focal points answer my questions"; and others prefer asked colleagues that they feel more secure or open to receive correct response. For example, one participant said that "I usually ask for help from people I think are more open". Thus, we can observe that despite trainings and content, newly PLs prefer ask for experienced colleagues. This scenario supports that we have to improve content to create best learning experience for newcomers.

Related to Wiki issues (Figure 2), we identified some problems that affect learning experience by newly PLs. Some PLs reported that improvements are related to usability issues. One participant said that "Someone give review before page published in wiki". Another participant had strong difficulty to add new resources in Wiki, and said that "I didn't find a way to write and add some HTML elements, like collapse sections. This report allows us identify need to add plugins or add-ons to support new resources and contents.

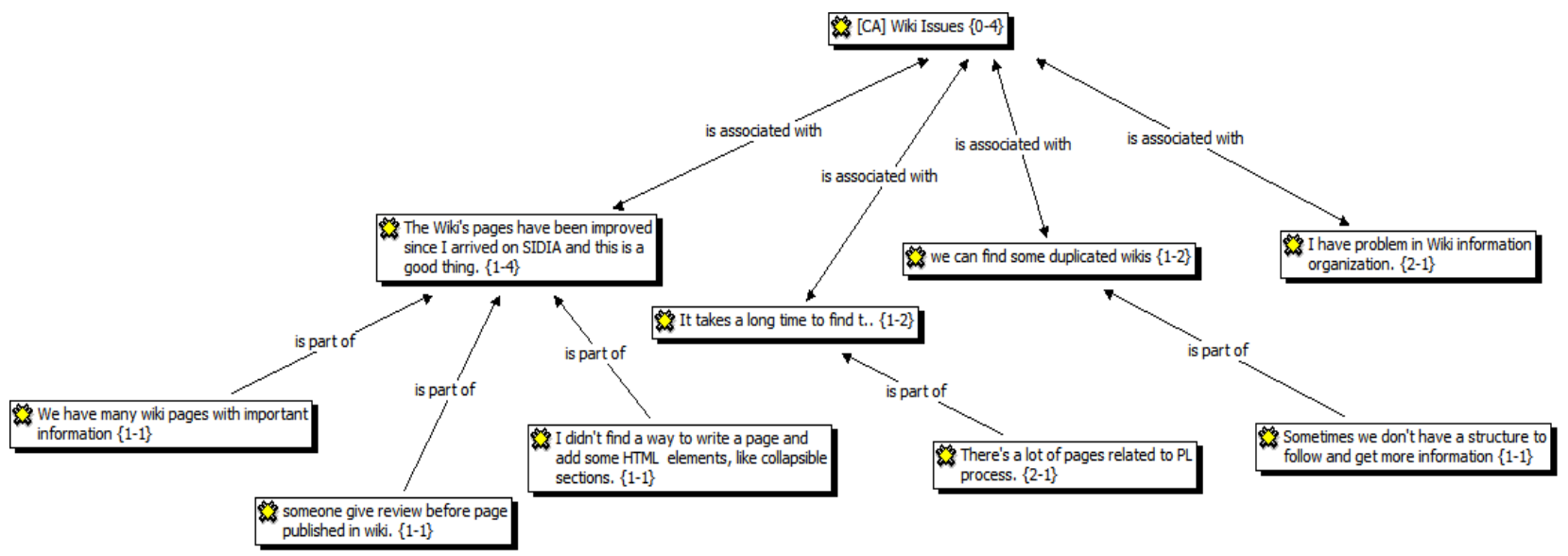

Figure 2. Wiki issues relations identified with qualitative analysis

Some participants reported that they found several pages duplicated as said one participant: "Sometimes we don't have structure to follow and get more information". Another participant said that "It's too difficult to find information on tool", caused by Wiki organization. These factors may assist on improving the user experience when fixed. Considering information issue, one participant said that "We have many pages with important information". These issues related usability and organization have been impacted negatively on PLs' interaction. In this case, these problems affect finding information desired by PLs.

Considering improvement and new ideas, most important contribution of this study was related usability improvements. These improvements are related to structural contents and topics suggested by subjects, see Figure 3.

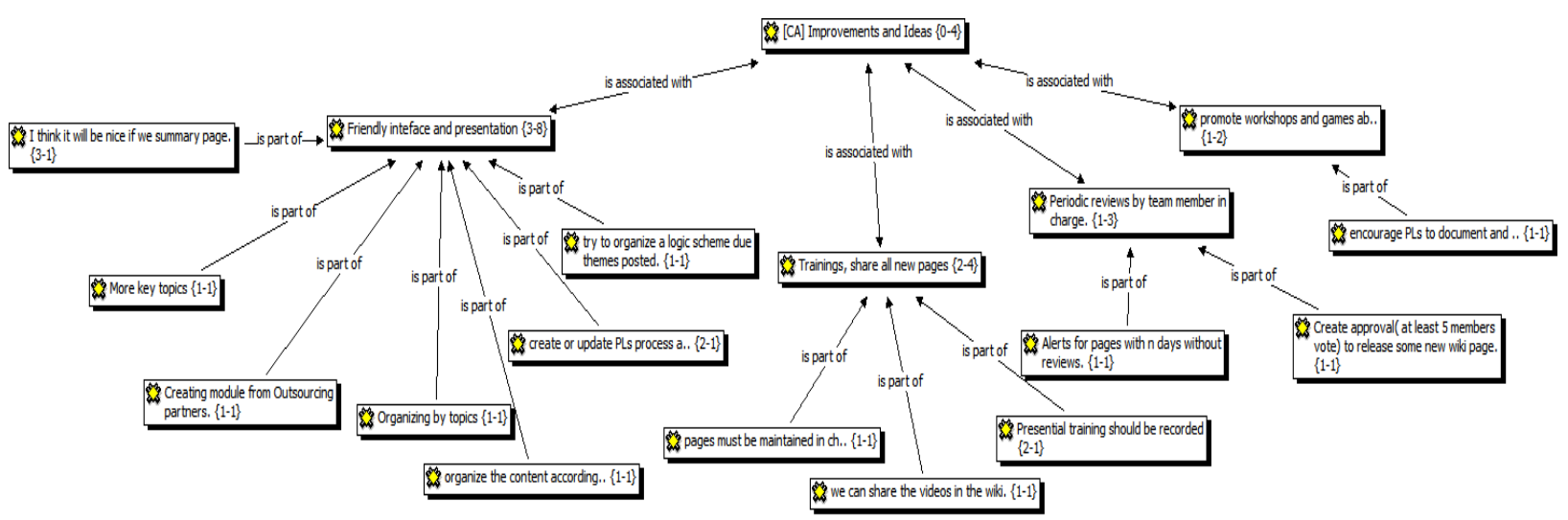

Figure 3. Improvements and ideas suggested by participants of study 
This qualitative analysis was important to improve Wiki design and to elicit new requirements to be developed. In this context, some participants reported as suggestion to include an index page to facilitate searches. One participant said that "I think it will be nice if we summary page". Other participant suggested "try to organize logic scheme due theme posted". One participant for example suggested: "More key topic" and "create or update PL process". In addition, some participants suggested to add new contents as online trainings, periodic review by specific team, alerts when pages have been updated and others improvements. One participant suggested "Presential training should be recorded" and "we can share video training in wiki". Other participant suggested to have games and workshops to share and involve PLs, said that "promote games and workshops". These findings were important to improve our training methods during integration phase of newly members. We use a tool to support record training, especially during pandemic period, that our team worked on home office scope. Next section presents contributions and new modifications developed after this case study.

\subsubsection{Study Contributions}

We used quantitative and qualitative results to improve Wiki content. Considering suggests gathered from participants' study, we created a backlog to better execution improvements. Main change was restructuring Wiki content presentation. Based on results we grouped information and contents based on common knowledge.

Initially Wiki was structured in one page, organized by topics. This organization caused strong difficult to find information. In this case, we created categories and grouped information as: processes, how-to and tutorials, useful information, organization links as showed Figure 4 below.

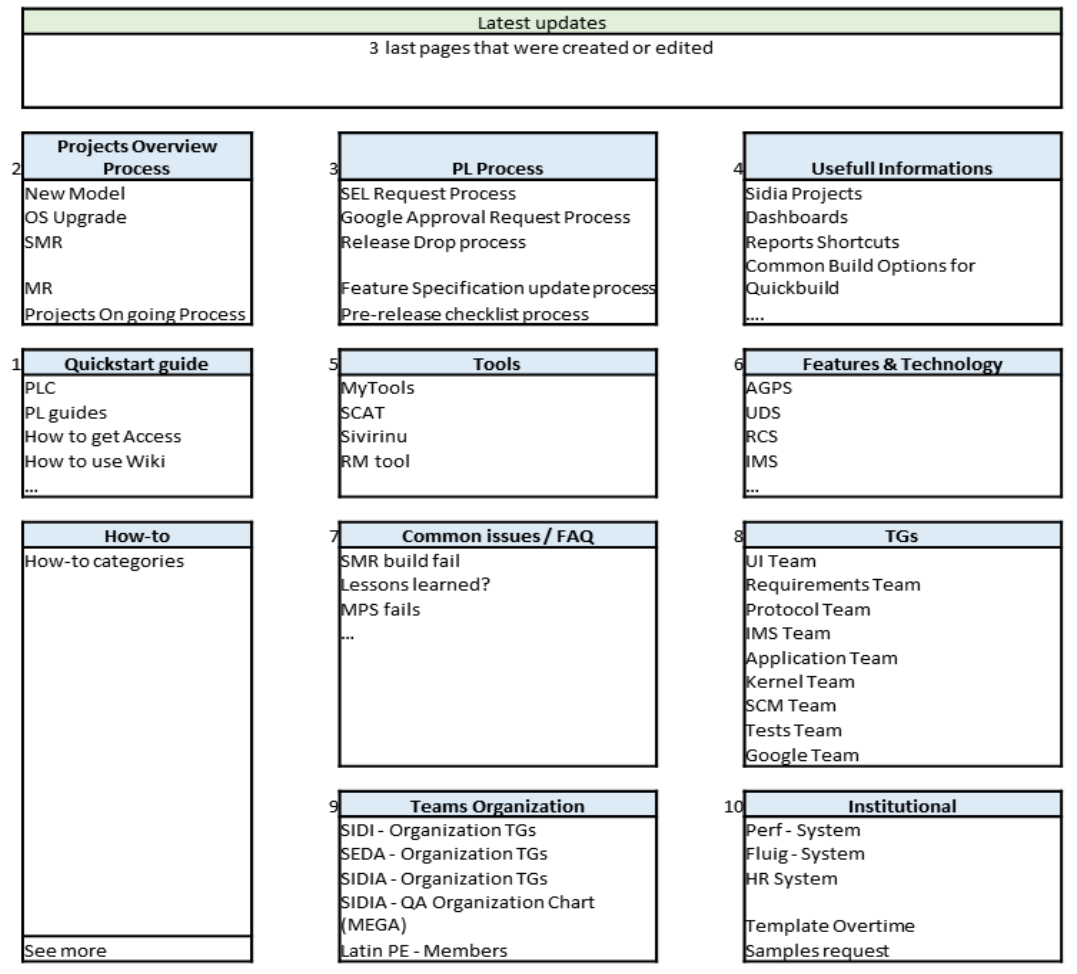

Figure 4. New Wiki structure organization

Figure 4 show new first organization approach adopted after of Wiki analysis. As main changes applied, we created sections based on common content aimed to improve learning during onboarding phase. In addition, we created index with order to better advice newly PLs which section order they should follow. Thus, we divided in chronological order for newly PLs. As improvement was created a specific group to evaluate changes and suggest changes. This group reorganize wiki organization and propose new order based on numbers (see Figure 4). In this case, first section is Project Overview, where newcomers can see all 
projects process executed by Sidia PL team. After that they can access specific process topics that they prefer, in section PL Process.

We also created common issues section. This section was created by experienced PLs with problems and project issues faced by them that can contribute to newly PLs. Other important change was how-to in specific section, and overview organization teams. As we work in GSD environment this section is important for PL know all team, know who is in charge for specific project (e.g. focal points, to assign issues etc.).

Another contribution was video training that we recorded and shared in specific Wiki page. In addition, we create a process to edit and share new Wiki pages. After this study, we have a specific team named as improvement process team, who is responsible to review and share new pages. We proposed also a template with good practices and content structure, following $5 \mathrm{~W} 2 \mathrm{H}$ method to facilitate learning, importance of each page and process related that page. The conclusion and future works are described on next Section.

\section{CONCLUSION}

This paper presented an extension study performed in Wiki, where we collected information about how newcomers learning about the working process at Sidia R\&D Institute. Previous work, described in detailed at Cunha et al. (2020), presented quantitative results and overview about difficulties faced by newly PLs during onboarding process at Sidia, specifically about knowledge transfer by new hires members. In this paper, we presented a qualitative analysis results and improvements made as contribution of this study.

In this sense, in previous work we conducted an empirical study attended by 24 volunteer newly PLs from Sidia as participants. We used an online questionnaire with questions based on TAM, considering ease of use, usefulness and motivation and engagement. As results presented, we have observed that participants have difficulty using the wiki, for instance, to search for information on the work process, nomenclatures, teams responsible etc. In this paper we conducted a qualitative analysis, using procedures of Grounded Theory to better understand difficulties and define actions to apply as improvements. In this case, we divide our findings into three categories: Wiki issues, Learning Strategies and Improvements and Ideas.

Considering Wiki issues, we observed that most issues are related to usability and structure content. As improvement we restructured content dividing in section by common knowledge. Considering Learning Strategies, we could see that newly PLs learn most effectively with experienced colleagues. In this case, we proposed training recorded and share in specific page. Considering Improvements, we create a team which is in charge of controlling and managing wiki content and we are planning new action as games and workshops to improve integration by newly PLs. Thus, promoting more contribution by these newcomers in Wiki content presentation.

Based on these results we are redesigning Wiki content. However, another interesting aspect to be investigated, as future work is how to minimize the impact of developer misunderstanding during integration in the company. In our case, we applied Wiki, but it is possible to recommend a set of data analytics based on developer profiles learning. However, it is an aspect that still needs further investigation.

Thus, we will replicate this study after improvement and compare the results with this work. We expect with this experience report to show through practical examples as it is possible to improve the learning process by newcomers in GSD environment. In addition, we intend to encourage software development industry to improve knowledge transfer to improve newcomers' onboarding to better support difficulties faced by them.

\section{ACKNOWLEDGEMENT}

Our thanks to Sidia team and SAMSUNG Electronics of Amazon Ltda for partial support, in the terms of Informatics Law $\mathrm{N}^{\circ} 8387 / 91$. 


\section{REFERENCES}

Avram, G. 2007. "Knowledge Wok Practices in Global Software Development," inECKM2007- Proceedings of the 8th European Conference on Knowledge Management, Barcelona, Spain, p 87-97.

Bao, L. et al., 2019. "A large scale study of long-time contributor prediction for GitHub projects," in: IEEE Transactions on Software Engineering, pp. $1-22$, Vol 1.

Darja Šmite N. B. M. A. Š. C. W., 2017. "Software teams and their knowledge networks in large-scale software development," Information and Software Technology, pp. 71 - 86.

E. Hustad, 2014. "Knowledge networking in global organizations: the transfer of knowledge," in: SIGMIS Conf. on Computer Personnel Research: Careers, Culture, and Ethics in A Networked Environment, 299-239.

F. S. K. D. a. E. A. G. Kanakis, 2019. "Supporting a flexible grouping mechanism for collaborating engineering teams," in: Proceedings of the 14th International Conference on Global Software Engineering.

G. Android, “Android Security Bulletin,” 1002 2020. [Online]. Available: https://source.android.com/security/bulletin.

Heredia, A. et al, 2017. "Tool-supported continuous business process innovation: a case study in globally distributed software teams," European J. International Management, pp. 388 - 405.

H. O. Barbosa et al. 2019. "Uma Análise do Uso de Ferramentas em Desenvolvimento Distribuído de Software para Atualização da Plataforma Android, " in IADIS Americana WWW/Internet 2019, pp. 29- 38. Portugal.

Kroll, J. Mäkiö J. and Assaad, M. 2016. "Challenges and Practices for Effective Knowledge Transfer in Globally Distributed Teams - A Systematic Literature Review," In: 8th International Joint Conference on Knowledge Discovery, Knowledge Engineering and Knowledge Management (IC3K 2016).

Mills, J., Ann, B., Karen, F. 2006. The development of constructivist grounded theory. In: International Journal of Qualitative methods. Cap 5, Vol. 1, 25-35.

Mahmood Niazi et al, 2015. "Empirical investigation of the challenges of the existing tools used in global software development projects," IET software, vol. Vol 9, p. 135 - 143.

M. Chuttur, 2009. "Overview of the Technology Acceptance Model: Origins, Developments and Future Directions," Sprouts: Working Papers on Information Systems, vol. 9, $\mathrm{n}^{\circ}$ Sprouts, pp. 9 - 37.

Portillo-Rodriguez et al., 2012. "Tools used in Global Software Engineering: A systematic mapping review," Information and Software Technology, vol. Vol 54, pp. 663 - 685. 